\title{
Kurdish Language Learning Tool in Serious Game
}

\author{
Gulala Ali Hama Amin \\ Database Technology Department \\ Technical College of Informatics \\ Sulaimani Polytechnic University \\ Sulaimani, Iraq \\ gulala.amin@spu.edu.iq
}

\author{
Karzan Hussein Sharif \\ Computer Science Department \\ College of Science and Technology \\ University of Human Development \\ Sulaimani, Iraq \\ karzan.hussein@uhd.edu.iq
}

\section{Article Info \\ Volume 5 - Issue 2 - \\ December 2020 \\ DOI: \\ 10.24017/science.2020.2.2 \\ Article history:}

Received : 13 August 2020

Accepted : 11 October 2020

\section{Keywords:}

Serious game, smartphone applications, Android language, Puzzle games. Application, Kurdish

\begin{abstract}
In this era, technology has a great impact on the educational system as they have on the other different areas of today's life, for instance, smartphone devices are the best examples. Software technology vendors moving toward developing tremendous applications in the education field. However, the lack of applications that satisfy the ambition of those wishing to learn the Kurdish language is still an obstacle in this field, specifically the field of smartphone devices technology and gaming industry. Hence smartphone devices become userfriendly and necessary tools in every individuals' life, as it is a multi-function device. In this paper we designed and implemented a smartphone application to learn the Kurdish language and improve vocabulary via a serious game. The application designed for android devices, enables users to learn and improve the Kurdish language. The application is also suitable for foreign people who desire to learn the Kurdish language. The system proposed in this paper introduces a smartphone application with an acceptable user interface for an exciting, interesting and challenging puzzle game at a time. The main goal is to learn Kurdish vocabulary using crossword puzzle, solving hidden words, find and circle the crossword in the puzzle solution in the context of a serious game.
\end{abstract}

\section{Copyright $@ 2020$ Kurdistan Journal of Applied Research.} All rights reserved.

\section{INTRODUCTION}

Languages are tools used to express feelings and thoughts to explore the world; it was the main feature for building a different civilization on the earth in the past. Words or language characters are fundamental elements of every languages discovered by a human being all over the history of civilization. Since language has significant importance in learning and educational systems, improving vocabulary knowledge is an educational priority in every 
process of learning a language including the basic language skills such as listening, speaking, reading, and writing [1].

The general concept that normally used with the word game is entertainment, this includes a variant of temperaments during gameplay, including happiness, enthusiasm, and feeling of satisfaction. However, if we look at the history of the development of smartphone applications, we discover that some of the application developers are addressed towards designing games for the purpose of education, not only just for entertainment. These types of games are called serious games. There are numbers of different methods to describe serious games. Some manufacturing brands consider serious games required to contain a veritable portion of gameplay paired with an applied characteristic. Whilst others use this concept with applications created with the use of software development from the videogame production, which signifies that a major part of simulators would be considered serious games. Further groups believe that serious games are basically videogames and that the classification is nothing more than a marketing technique. For the purposes of this study, the following definition has been utilized: serious games are applications that combine aspects of tutoring, teaching, and training, with an entertainment mode. With these features, the application's goal will be conveniently reflected as an application that contains serious and entertaining content at the same time [2]. From the previous definition, it can be said that serious games are formed and used to function for a reason or a target, rather than pure entertainment. For instance, provide skills, increase the level of knowledge and learning outcome for which they have been created [1]. Previously some evidences had been found on several games proved that have advantageous in academic achievement purposes, furthermore, games can essentially be integrated within a classroom to improve student learning and contribution [3]. Serious games are considered the best choice for various fields of life, for instance, teaching and training, information and communication, health, advertising, etc. [2]. Using a serious game to learn children with special disorders, for instance, children with autism confirmed that learning of vocabulary items among children with autism enhanced significantly when they experience this category of the game [4],[5]. Moreover, physical activities using a serious game for children with a chronic disease may have some positive impact on the children to promote physical activity levels among these children [2].

The rest of this paper consist of a literature review, contribution, serious games, methodology, results, and the last part is a conclusion and future works.

\section{LITERATURE REVIEW}

There are many studies focused on the serious game in the educational systems. In [6], the authors in their research work developed a serious game named Kockica which is a 3dimension serious game has been developed in the Bosnian native language to help children with disabilities; neuro-typical children as a special case, in order to improve their verbal skillfulness and learn the alphabet in an entertaining and fascinating way. However the benefit of this game depends on the level of contribution of parents and teachers who should help the children with disabilities to achieve the serious games' goals. While authors in [7], additionally targeted different learning goals with the Bosnian language, they motivated the same category of children to engage with a serious game application developed to learn counting and color matching, however, the application limited to learn children only one area or scope (airport) in the serious game content. Darwesh et al selected Kurdish Sorani dialect and Arami alphabet of Kurdish language to create a serious game for the range of children between ages 4-12 years old, they proposed hypotheses for a serious game and designed a prototype to test the hypothesis focusing on writing approach, hence they just conducted a survey and interviewed pupils and teachers to collect information about what features they need to add in their hypothesis to ensure that they target the main goals behind the proposed system [8]. 
Another example of a smartphone application related to learning a specific language is for the Japanese language, the study in [9] used a technic named shadowing. This technic is famous in Japan supports learner to learn Japanese language by hearing a recorded speech and the learner try to pronounce a word or sentence correctly, and repeat the process as much as possible to reach a perfect level. Shadowing technique is assists to learn new vocabularies as well as improving speaking skill which is mostly not included in Japanese language curriculum school, since the Japanese Language Proficiency Test (JLPT) exam materials lack of this skill test. Moreover, pronunciation in the Japanese Language is considered to be one of the reasons that make students face difficulties to speak Japanese in a fluent way. Practicing the traditional shadowing technic without assistance is boring, excepting less motivation, and lack of feedback from instructor whether the pronunciation is correct or incorrect. Thus, using this gamification, "Shadowingu app" is confirmed to be efficient learning tool. Since the study implemented gamification elements that can be applied such as progression, rewards, leaderboard, etc., all these elements provide a learning environment that engage student to achieve better result. However, this study has not yet examined whether the effects of this gamification application similar over long-term period or only due to its novelty. It needs further research take into consideration learning materials like Kanji, grammar for Japanese Language, and writing skills to students stay active.

The English language is in demand and popular to a large degree, also considered a global one among the spoken languages in recent decades, therefore, studies [10], [11], [12] related to this language. In China, Authors in [10], stated that, undergraduate students lack adequate learning plan when they intended to learn and practice new vocabularies in English. For this reason they proposed in their study a game application named "Hujiang Happy Word Games" application to examine whether their technic of joyful learning services in this context. They observed that this joyful and pleasurable learning can improve students' curiosity and selfconfidence of learning, however, no significant effects on vocabulary learning has been observed, since it cannot boost the learning effects of English vocabulary process of learning as compared to the traditional ways. Indeed, still some limitations in this research are exist, for instance, the scope of learning tools' selection is pretty limited, the participants are consist of only 24 students from a University in Beijing City, another limitation is the time for the participants to learn is quite short which may affect the result of the research work [10].

Hanandeh et al, in their paper work examined the effects of serious game performance and learning tasks based on a model using technology in pedagogical content knowledge frame on students' motivation to English reading. A pre-experimental study was conducted amongst 50 undergraduate students at Yarmouk University, Jordan for semester II, 2016-2017. Furthermore, pre and post motivation questionnaires had been used as a measurement to the students' motivation for English reading skill. As a result they indicated that there is a significant improvement observed in the level of motivation towards learning English reading skill. Moreover, the learning tasks had a great effect on students' interest, pleasure, confidence and satisfaction scale. A serious game activity and learning tasks based on ARCS model within the technological pedagogical content knowledge (TPACK) framework were utilized to increase motivation of students in reading English. The results showed advantages of the work. However, it has been noticed that there is a lack of the experimental studies that investigate SGs effectiveness in learning, specifically in English language learning. Thus, more researches should be conducted to explore serious games influence in English language skills such as reading skill [11].

The study in [12], is a preliminary experimental study, provides a taxonomy of the outstanding opportunities and challenges as a result of using serious games to learn the English language. The Delphi method had been used to collect students' opinions in computer science department and resulting from a SWOT analysis to use these two different gamebased methods of learning English. This study produced a well-organized list of important adoption and entry issues to consider when using the English language learning knowledge. Additional factors were identified through discussion of these issues. Based on this, the importance and implications of opportunities and challenges associated with serious games 
has been highlighted. Furthermore, the study listed many important advantages and prospects that promising to use serious games in classrooms. However, it acknowledged many drawbacks that should be take into account to be controlled.

\section{CONTRIBUTION}

The evolution of the educational serious game leaves plenty to be wanted especially during the current time as the whole world suffers from an outbreak of the epidemic due to the Corona virus (Covid-19) where learners and teachers are away from physical facilities and cannot attend actual classes especially in Kurdistan where education establishments stopped during Corona epidemic. So, we designed, developed, and implemented a smartphone application to learn the Kurdish language and improve vocabulary via a serious game application designed for android devices, enables the user to learn and improve the Kurdish language. The application is also suitable for foreign people who want to learn the Kurdish language. It is worth mentioning that, our proposed system is unique and original since there is no evidence on existing a similar serious game focusing on improving the Kurdish language through learning vocabulary, with a user-friendly interface and challenging levels.

\section{SERIOUS GAMES}

In recent decades, smartphone technological industries left their mark in developing applications for emerging learners in a real context. Mobile technologies in a specific serious games subsection called learning games support more appealing learning involvements. From this context, serious games are underlined as games used for pedagogical purposes rather than mere entertainment. This might be a reason that the next generation will integrate more involved and shared games scenarios that will increase the learners' inspiration [13]. Some studies show that languages can be learned via serious games, others introduced specific topics in computer science; for instance, programing language in the field of computer science is a suitable module to be learned by a serious game, similarly learning languages is one of the best examples [8], [14], [15].

The concept of "Serious Games" used for the first time by Clark C. Abt, in a book in 1970, holding the same title. A serious game is a form of a game whose main objective is not entertaining the user nevertheless learning. They are organizing intellectual competition with specific game rules that use common sense entertainment in a serious position to meet educational goals, regardless of pedagogical context [16]. The serious game branch adopts an improved framework and provides behavioral simulation, users learning, and eventually becomes an effective feedback route and then substantially changes the learning experiences [16], [17].

The main purpose of a serious game is to provide the background for user participation in specific learning activities and to create a motivating atmosphere. In other quarters, a particular serious game designer believes that the user should be involved in the flow of learning processes and, eventually, in highly permitted activities. Serious games have been applied extensively to increase the swiftness of participation in learning and raise the amount of motivation to support the improvement of related intellectual skills. In the serious game, the user will explore contextual concepts and links in a specific educational context and asked to explain them and finally understand them [16], [18].

Serious game application as a method is based on trial and error, users can improve collaboration, social leadership skills and cooperation by making mistakes in a controlled environment. The most important principle related to the serious game is the use of the internal drive to improve the learning process; in this sense, the conceptual structure of the game as a mental and physical motivation can eventually compel users to make decisions, choose, rank, provide solutions, etc. Ultimately, users can improve social and practical skills to create the game. The serious game environment will provide stimulation support through self-learning skills for players "players often pursue knowledge to control game flow" and involve their natural senses; it allows the user to extract the value by learning from existing 
facts or even under predetermined simulation conditions. It can be said that serious game is a strategic step on the way to affirmative interaction by the user. The advantage of a serious game learning methodology as a strategy lies in its ability to provide content relevant to specific educational goals, rather than absorbing fun games or leisure games that already exist within common educational practices [16], [17].

\section{METHODOLOGY}

For the proposed system methodologies is the best choice to come up with proper process for designing, implementing and testing the serious game to avoid the gap which has been determined in the literature review, resulting in creating a smartphone game application with the different target specified for the Kurdish language. Our methodology for developing of the game application followed 5 stages, namely: concept, design, assembly, development and making evaluation and testing. The methodologies procedure in this research adaptation is explained in details as follow:

\subsection{Game Concept}

The concept of the proposed game is designing and implementing a smartphone application to learn the Kurdish language and improve vocabulary via a serious game application designed for android devices, enables the user to learn and improve the Kurdish language. Additionally, the application is suitable for foreign people who have a passion to learn the Kurdish language. It is essential to develop learning education tools particularly, multimedia in an attractive and convenient environment that is appropriate to the characteristics of students or users and modified according to their needs, packaged in the form of Android-based game applications that are more functional and effective.

\subsection{Game Design Process}

Before the development of any game, there are a number of mechanisms in the design process that should be done. The most important of our game design process is the Game Design Elements and Flowchart of the game.

\subsubsection{Game Architecture Diagram}

The game architecture diagram clearly shows the architecture of the Game. The User Interface used for interaction with the logic of the application. The word search game gets the data from the database which is SQLite in the application. This Interacts and gives the data to the application logic and the information is processed and displayed back on the user interface.

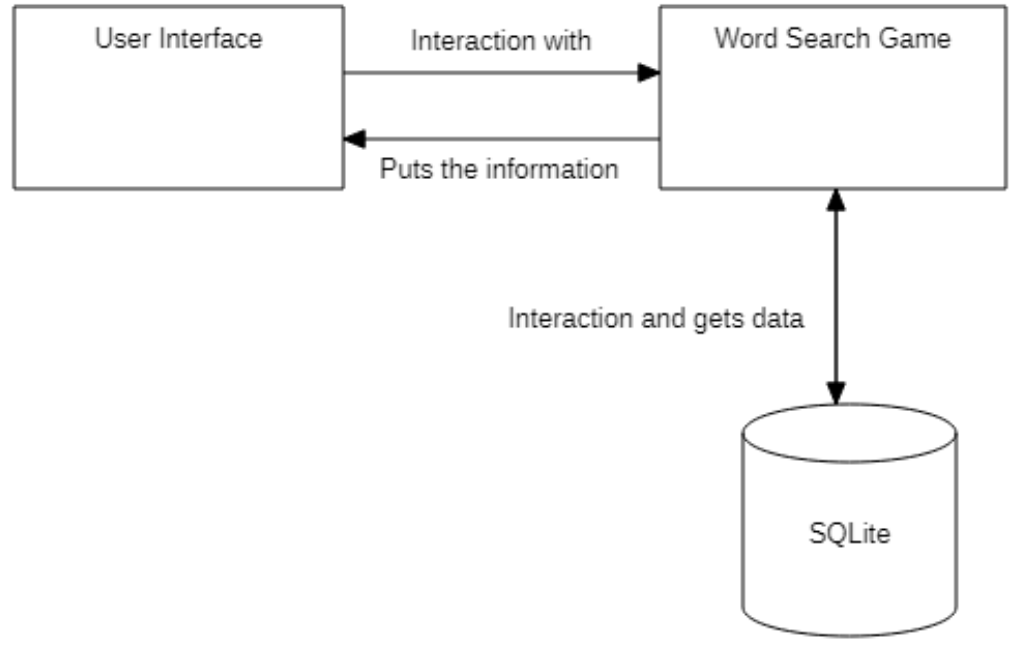

Figure 1: General Game Architecture 


\subsubsection{Game Design elements}

The game framework consists of words, learning through this set of words and searching, for instance, a word puzzle game. On average the gameplay test practice rely on how completely the system design components are taken into account to improve user participation. For the game to function appropriately, some elements such as words and finding results are required. Regardless, the needs of the player/user to control and verify the environment and outlines, and most significantly, interacts throughout a convenient interface. If we map the design elements to the gaming condition and environment, finding a word and output the word one by one are the game technique that leads the game pattern to a remarkable approach and makes users reach a level of satisfaction. The procedure that occurs in the internal system is not required for the user to experience the gameplay. These game dynamics are occurring in the background and generally unnoticed. These invisible elements constitute the game dynamics that are firmly required for the proper operation of the game. Game developers should continuously be aware of the ambition and interest of the user and how elements are designed in the game. Furthermore, the game inventor capable of utilizing game technics that tolerate and excess motivation of the user. The game quality is a significant portion that surprises the user in getting them fascinated with and lunch into the game.

The following subsections explain features about our game elements that make the user keep playing without boring and making the gamer/user passionate about the game:

\subsubsection{Rules}

The game rules require to be distinct and compulsory for playerslusers in order to trust the game and comprehend that they are playing a variety of fair game. Moreover, they need to be informed precisely what leads them to win in order to keep involved and to carry on with the game.

\subsubsection{Levels}

Levels indicate the accomplishment of the partition targets of the game. Each level presents the users progress throughout the game. Our game application has a set of levels that each player in-game has a collection of unorganized words in each level to play representing the level of the game.

\subsubsection{Time pressure}

Time refers to how the period of time specified for each level is controlled. The game designer can choose to use time in a variety of ways. In our game, the timer starts when the user lunches the game level as should be, along with setting an alarm regarding the amount of time remaining or spend to finishing this level creates a sort of challenging and inspiring atmosphere for the user during the gameplay.

\subsubsection{Points}

The game needs a process of giving scores to the player. Thus points are a crucial and essential element in the game to determine and track users' progress. With points, users are permitted to claim rewards or even use them to unlock a higher level as a result of achievement steps.

\subsubsection{Leaderboard}

The leaderboard is the game element that illustrates the accomplishment and individual performance of a user. Moreover, this element reflects users' hard work and self-affirmation.

5.2.2.6 Achievements

This element shows the player's development and achievement during the game.

\subsubsection{Game Design Flowcharts}

In order to clarify some of the most complicated sections of the game, we need to explain not just the mechanics, but also how the game's objects interact with each other, what your game is about, and how it looks and works. This can be achieved by using flowchart, which is graphical representation of algorithm, workflow or process.

\subsubsection{General Flowchart of the proposed app}

When the application starts, it reads the user input which can be select one pf these options: level to play, Settings and Information about how to play and about the application. If user 
select "select level to play" then for each level, the user will have three options to choose i.e. Easy, Medium and Hard. If user selects "settings" then user can change some setting of the application. If user select "Information about how to play an about the application" then user can find information about the application and how the application works by play video. The General Flowchart of the proposed application is presented in the Figure 2

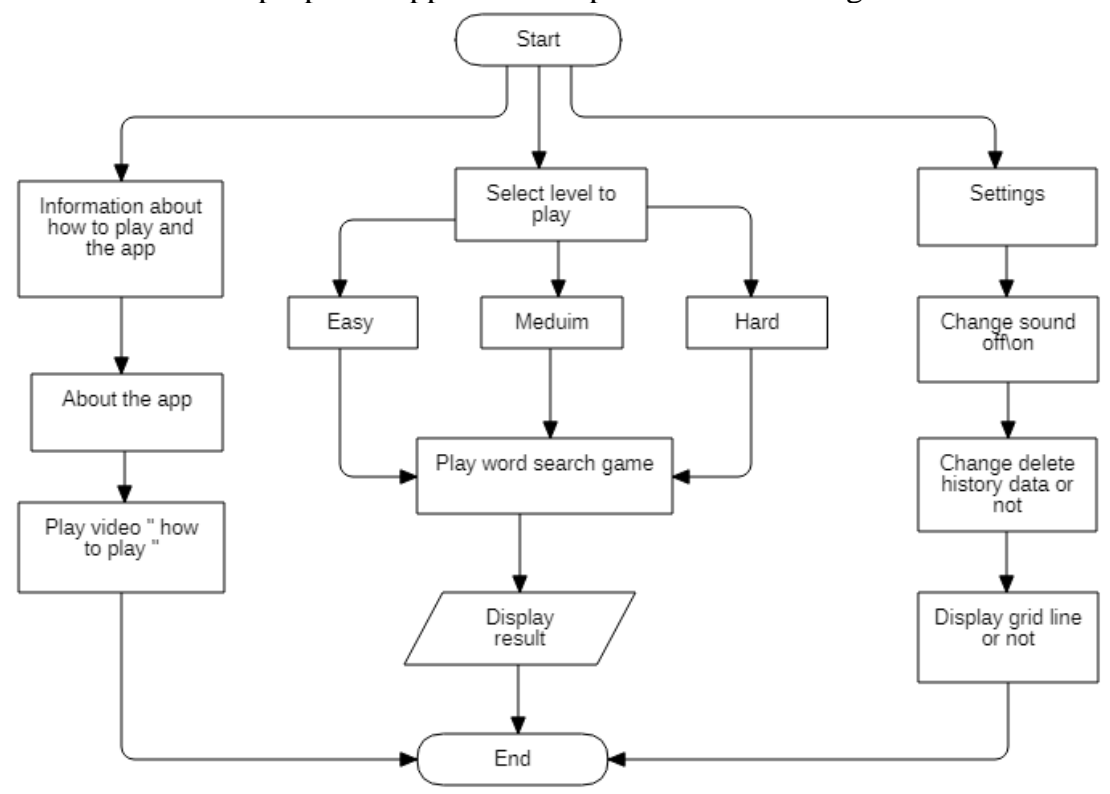

Figure 2: General Flowchart of the proposed app

\subsubsection{General flowcharts of the game}

The process of scrambling words is done by filling the matrix with the words you are looking for according to the rules, after the search found then storing the coordinates of that word will be saved. For more details, following are some flowcharts that clarify most complicated sections of the game in Figures (3, 4 and 5).

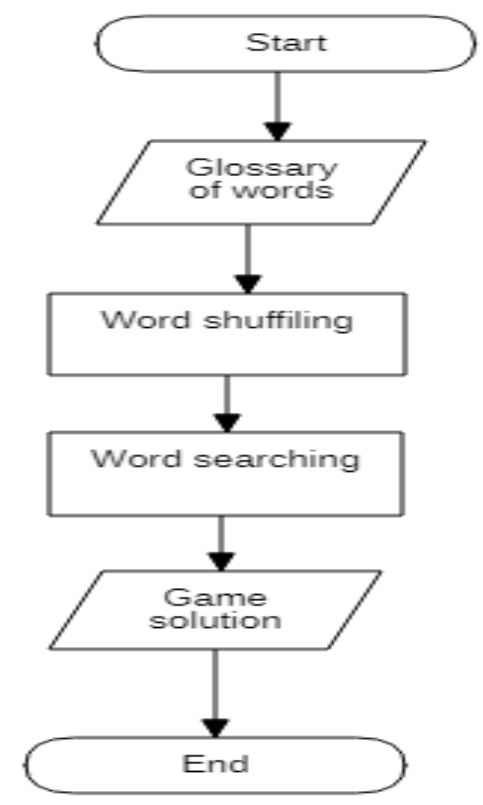

Figure 3: General flowcharts of word search game 


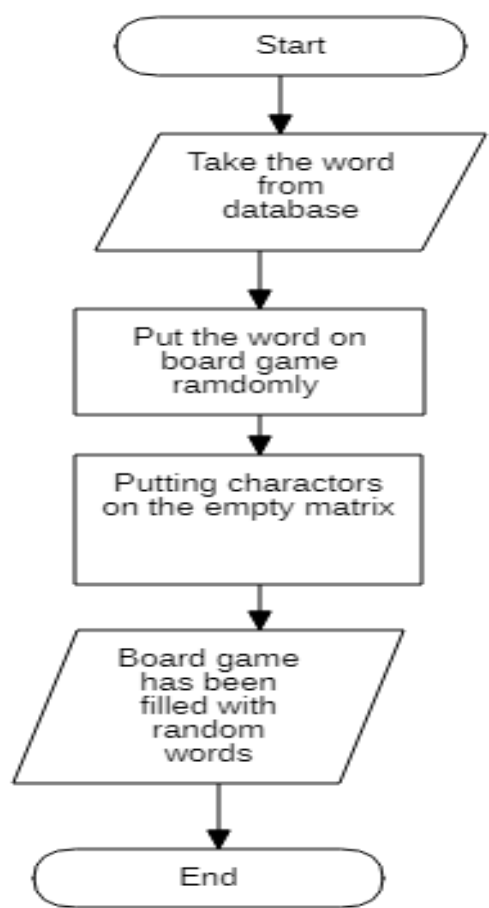

Figure 4: Flowchart of the word shuffling

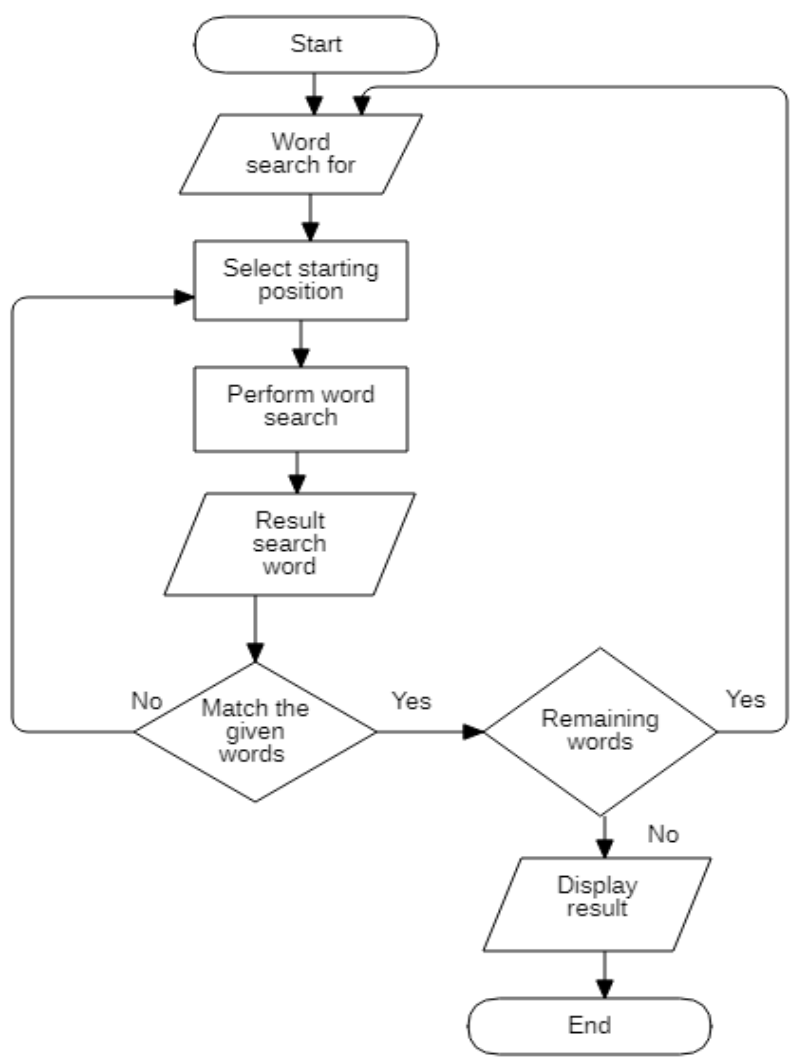

Figure 5: Flowchart of word search 


\subsection{Assembly}

Assembly is the stage of making all objects or media game materials, based on flowcharts, and navigation structures that come from the design stage leads to creating applications.

\subsection{Game Development}

It is worth mentioning that our game application is developed for Android devices, in other words is an Android-based application that has been created using Android Studio IDE and java programming language, Adobe illustrator used for making all of design include; background, buttons and all other design in our application. From the levels (Easy, Medium, and Hard), the player can decide on which level to start the game. And there is a help button that can instruct the player step-by-step procedure and the application content rules (How to play), Leaderboard, Timer, Points, and other elements that are explained in the previous section. The game has the correct environment made for the (Kurdish Language Learning Tool), it has all the specifications that should be present in an actual serious game.

\subsection{Game Test}

After the assembly phase and development phase completed, the testing phase is done by running the application/program to check and observe errors then find solutions to ensure that the product completed is as desired. Our game application was tested with all android versions including android 10.0 Q and below. Also, it successfully tested with all devices that run android smartphones and tablets with different screen sizes of the devices that work with the android platform. Android Studio is designed to make testing simple. Our game application was tested by using instrumented tests. Android Studio provides source code directories (source sets), for our test. In this type of testing the application runs on a hardware device or emulator. We used both of them emulator and real device by connecting to the android studio IDE.

\section{RESULTS}

In this section we show some of the features in the game application, which is consist of a user interface, once the user clicks on the game icon of the application a splash screen will be displayed, as shown in figure (6):

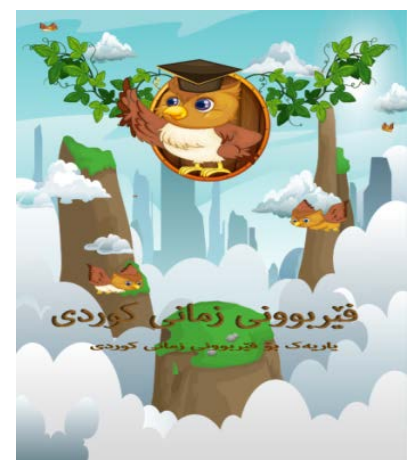

Figure 6: Splash screen of the application

The screen splash shows a quick idea about the game after that the application directs you to the home-screen as shown in figure (7) below, the figure illustrates that the screen is consists of several buttons; (New game), (History), ( How to play ), (About) and (Settings): 


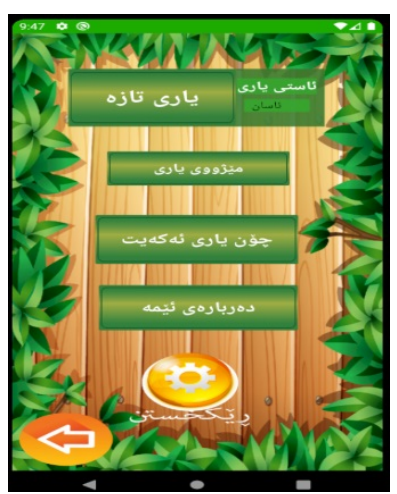

Figure 7: Home screen of the application

When you start a new game, you have to decide the level of the game, the game has three levels (Easy, Medium, Hard), click on the (new game) button a drop-down menu displayed, you have to click on the level you want and start, figure (8) below shows that:

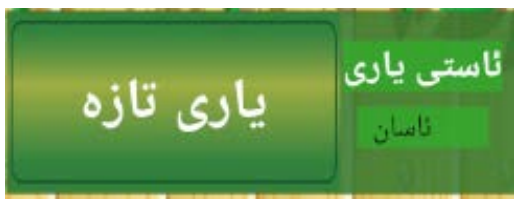

Figure 8: Starting a new game

The levels of the game indicate that the more your background about the Kurdish language is strong the more you can make progress in improving your Kurdish language level, in figure (9) the levels of difficulty of the game are displayed.

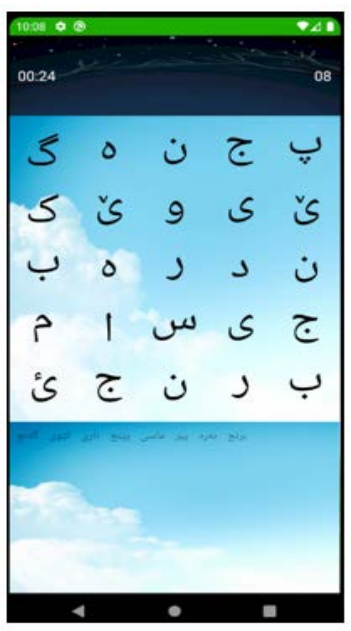

(a) Easy

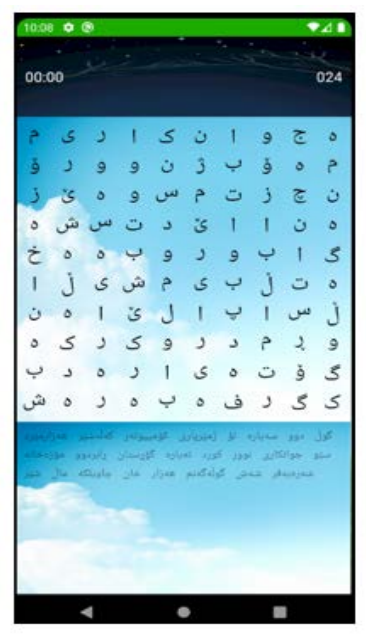

(b) Medium

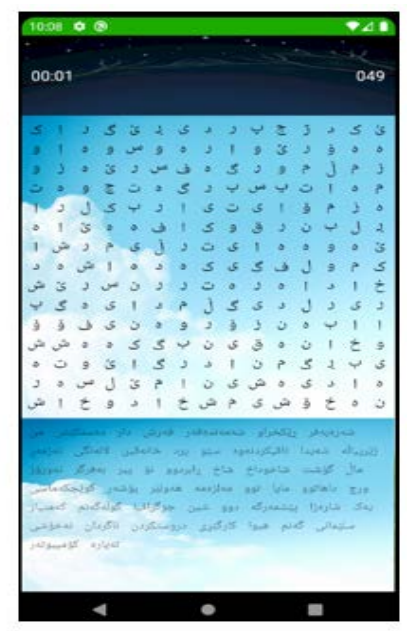

(c) Hard

Figure 9: Levels of difficulties of the game

\section{CONCLUSION AND FUTURE WORKS}

This research paper presents a novel game application for smartphone devices; the application is a serious game in order to help users to learn the Kurdish language through an application designed for Android devices. It is obvious that game programming taking a great deal recently in the field of mobile programming, since the later has become an effective tool that 
could be used in various purposes, educational purposes are one of these purposes. In this work, once the users install the application on the device; can be played at home, in the classroom, during a trip, etc. When the users use this application to learn the Kurdish language, their aspiration increases as they have to challenge their selves to pass all the threelevels of the game. Future versions of this game might cover a specific Kurdish language module in high schools or primary schools in Kurdistan region in Iraq, to help students to cover the topics of the module and assists the instructors as well. Also future step involves in creating a story plot pattern to be used as a tool for facilitating users delve deeper in the more complex level of the Kurdish language. The latter can encourage foreigners and be a good incentive for those who have strong aspirations to learn this language without the help of a facilitator, install the application on their smartphones and enjoy learning Kurdish

\section{REFERENCE}

[1] A. Sukstrienwong and P. Vongsumedh, "Software development of word search game on smart phones in English vocabulary learning. In The Proceedings of the International Conference on Education and Modern Educational Technologies, Vol. 39, pp. 37-42, 2013.

[2] J. Alvarez and L. Michaud, "Serious games - Advergaming, edugaming, training and more ...” [Online]. Available: http://ja.games.free.fr/ludoscience/PDF/EtudeIDATE08_UK.pdf. [Accessed: 05-Jul-2020].

[3] N. M.nazry and D. M. Romano, "Mood and learning in navigation-based serious games,” Computers in Human Behavior, vol. 73, pp. 596-604, 2017.

[4] K. Khowaja and S. S. Salim, "Serious Game for Children with Autism to Learn Vocabulary: An Experimental Evaluation,” International Journal of Human-Computer Interaction, vol. 35, no. 1, pp. 1-26, 2018.

[5] D. Bossen, A. Broekema, B. Visser, A. Brons, A. Timmerman, F. V. Etten-Jamaludin, K. Braam, and R. Engelbert, "Effectiveness of Serious Games to Increase Physical Activity in Children With a Chronic Disease: Systematic Review With Meta-Analysis,” Journal of Medical Internet Research, vol. 22, no. 4, 2020.

[6] S. Bajraktarević and B. Ramić-Brkić, "Kockica: Developing a Serious Game for Alphabet Learning and Practising Vocabulary," Lecture Notes in Networks and Systems Advanced Technologies, Systems, and Applications, pp. 349-358, 2016.

[7] A. Kolaković and B. Ramić-Brkić, “Aviončići: Developing a Serious Game for Counting and Color-Matching,” Lecture Notes in Networks and Systems Advanced Technologies, Systems, and Applications, pp. 359-367, 2016.

[8] A. M. Darwesh, M. I. Ghareb, and S. Karimi, "Towards a Serious Game for Kurdish Language Learning," Journal of University of Human Development, vol. 1, no. 3, p. 376, 2015.

[9] H. C. Kurniawan, B. Sitohang, and S. A. Rukmono, "Gamification of Mobile-based Japanese Language Shadowing,” 2019 International Conference of Artificial Intelligence and Information Technology (ICAIIT), 2019.

[10] J. He, Y. Lee, B. Young, and F.-K. Chiang, “A Study on the Effect of Joyful Learning Application upon Undergraduate English Vocabulary Learning,” 2017 International Conference of Educational Innovation through Technology (EITT), 2017.

[11] A. Hanandeh, Z. Abdullah, and J. Harun, "The Effects of A Serious Game Activity and Learning Tasks on Students' Motivation Towards Reading Skill,” 2018 IEEE 10th International Conference on Engineering Education (ICEED), 2018.

[12] N. Yanes and I. Bououd, “Using Gamification and Serious Games for English Language Learning,” 2019 International Conference on Computer and Information Sciences (ICCIS), 2019.

[13] S. George and A. Serna, "Introducing Mobility in Serious Games: Enhancing Situated and Collaborative Learning,” Lecture Notes in Computer Science Human-Computer Interaction. Users and Applications, pp. 12$20,2011$.

[14] K. Nakajima and T. Nakajima, “A Vocabulary Learning Game Using a Serious-Game Approach,” Lecture Notes in Electrical Engineering Advanced Technologies, Embedded and Multimedia for Human-centric Computing, pp. 13-22, 2013.

[15] A. Yassine, D. Chenouni, M. Berrada, and A. Tahiri, “A Serious Game for Learning C Programming Language Concepts Using Solo Taxonomy,” International Journal of Emerging Technologies in Learning (iJET), vol. 12, no. 03, p. 110, 2017.

[16] B. H. Yousefi and H. Mirkhezri, “Toward A Game-based Learning Platform: A Comparative Conceptual Framework for Serious Games,” 2019 International Serious Games Symposium (ISGS), 2019.

[17] A. D. Gloria, F. Bellotti, and R. Berta, "Serious Games for education and training," International Journal of Serious Games, vol. 1, no. 1, 2014.

[18] M. A. Camilleri and A. C. Camilleri, “Student-Centred Learning Through Serious Games,” INTED2019 Proceedings, 2019. 